\title{
THE FIRST HEART SOUND IN AURICULAR AND VENTRICULAR EXTRASYSTOLES
}

BY

\author{
PEDRO COSSIO, RICARDO G. DAMBROSI, AND H. F. WARNFORD-THOMSON \\ From the Cardiological Department, Semiological Institute, Buenos Aires, and the Cardiovascular Clinic, \\ British Hospital, Buenos Aires \\ Received December 21; 1946
}

It is well known that the first heart sound of a premature beat may be of greater or lesser intensity than the first sound of the preceding or following normal beats (Cossio, 1944). An increased intensity of the extrasystolic first sound might be due to the onset of a premature ventricular beat coinciding with the auricular systole of sinus origin (Lewis, 1935), but this explanation could only apply to an extrasystole of ventricular type. As Cossio and others $(1943,1944,1945)$ have shown that an increased intensity of the first sound depends on the position and tension of the auriculo-ventricular valves at the onset of ventricular systole, it was decided to investigate the behaviour of the first sound in premature beats and to determine its clinical significance.

\section{Present Investigation}

Simultaneous electrocardiographic and phonocardiographic records, with a camera speed of $75 \mathrm{~mm}$. a second, were obtained from 30 patients with extrasystoles. First, the auricular or ventricular origin of the premature beats, and their situation in diastole, that is their degree of prematurity, were established; and also the time relation of the ventricular systole of the premature beat to the premature auricular systole in the case of auricular extrasystoles, and to the auricular systole of sinus origin in the case of ventricular extrasystoles. Secondly, the intensity of the first sound of the premature beat was determined by phonocardiogram and compared with that of the preceding and following normal beats. The time interval between the onset of ventricular systole and the first sound (onset of QRS to first sound) was determined both in the premature and the normal beats.

Of the 30 cases investigated, 16 proved to have auricular and 14 ventricular extrasystoles.

\section{Auricular EXTRASYSTOLES}

In all but one of the 16 cases (Table I), the extrasystolic first sound was always louder than the first sound of the preceding and following normal beats. The time interval between the onset of QRS and the reinforced extrasystolic first sound varied from 0.05 to 0.08 seconds, while in the case of normal beats or premature beats without reinforcement of the first sound, it was 0.03 to 0.05 seconds. Thus in relation to QRS, the reinforced extrasystolic first sound occurred from 0.01 to 0.05 seconds, average $0.03^{\circ}$ seconds, later than the first sound of normal beats.

The increased intensity of the extrasystolic first sound and its corresponding delay bear a close relation to its degree of prematurity. The greatest intensity and delay of the first sound was observed when the extrasystolic ventricular systole occurred some time after the $T$ wave of the preceding cycle; that is in mid-diastole (Fig. 1). In contrast, the first sound was less intense, even less than that of normal beats, when the extrasystolic ventricular systole occurred 


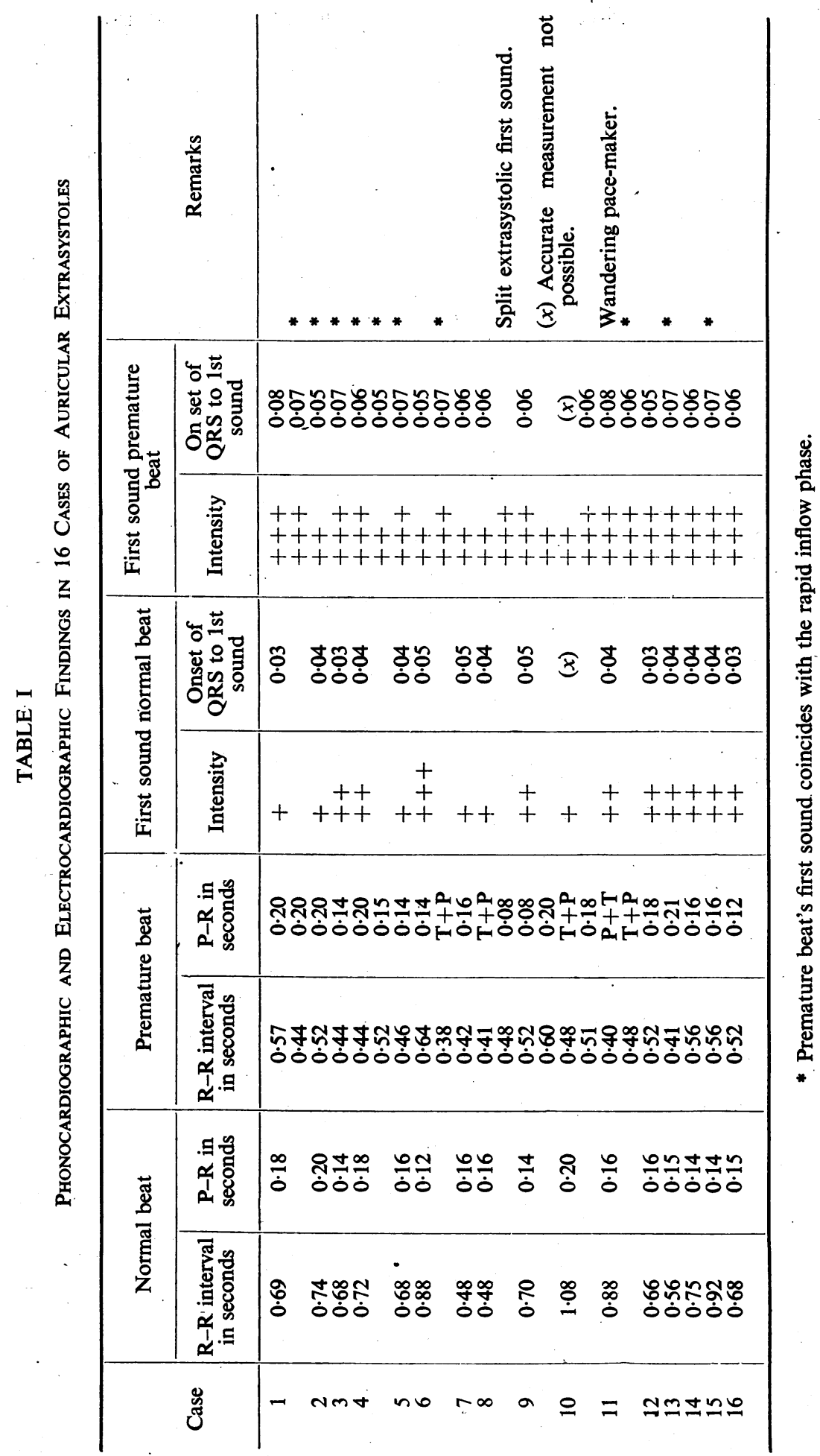




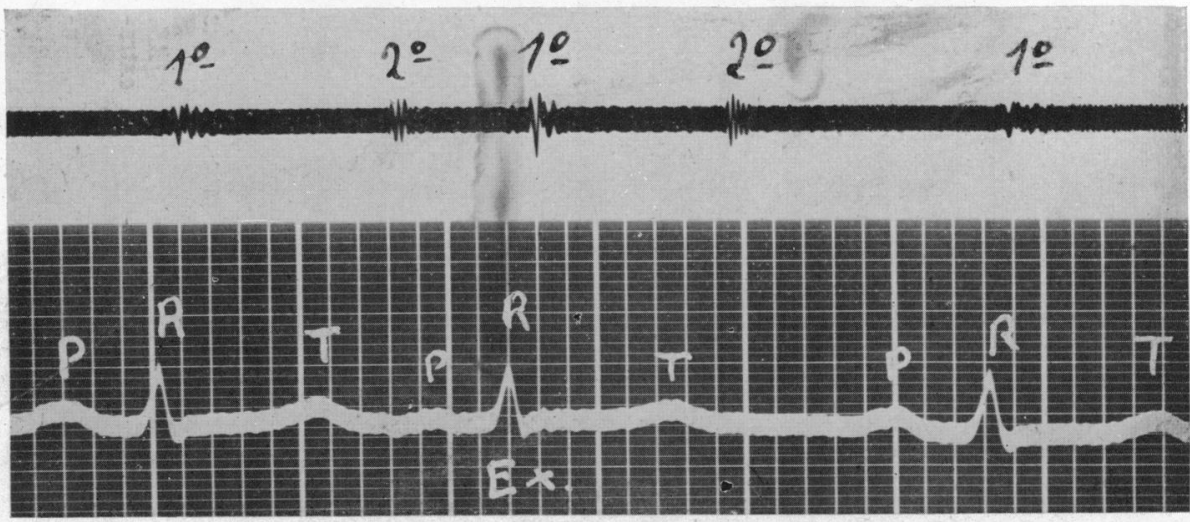

FIG. 1.-Simultaneous phonocardiogram and electrocardiogram showing auricular premature beat falling in mid-diastole; the first sound is intensified and delayed.

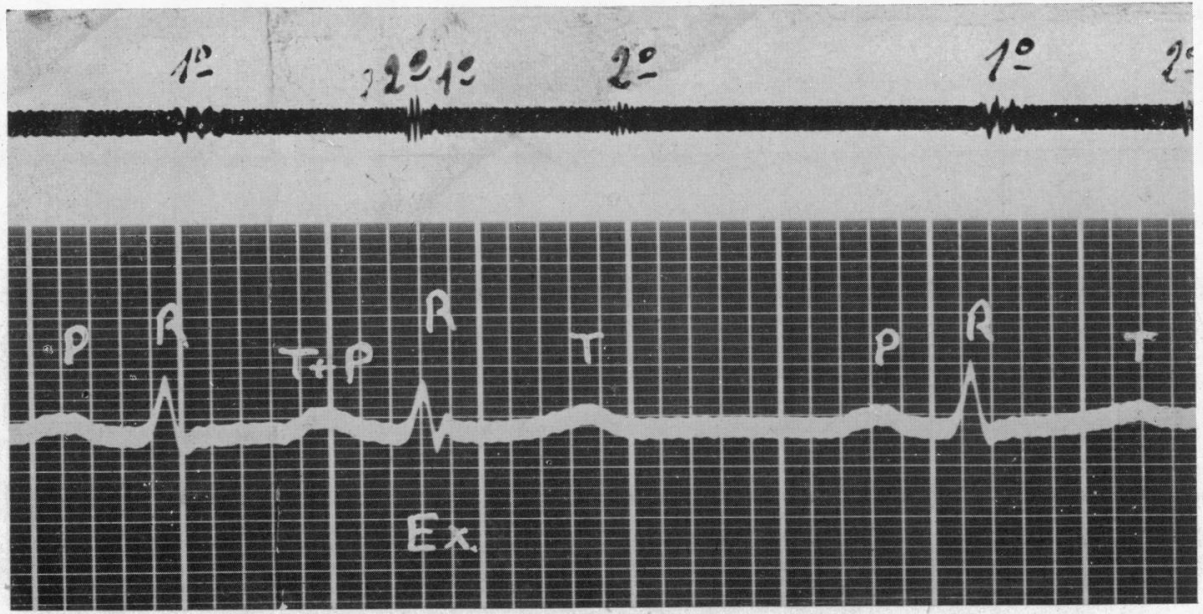

FIG. 2.-Auricular premature beat occurring early in diastole, with a fainter first sound than that of normal beats.

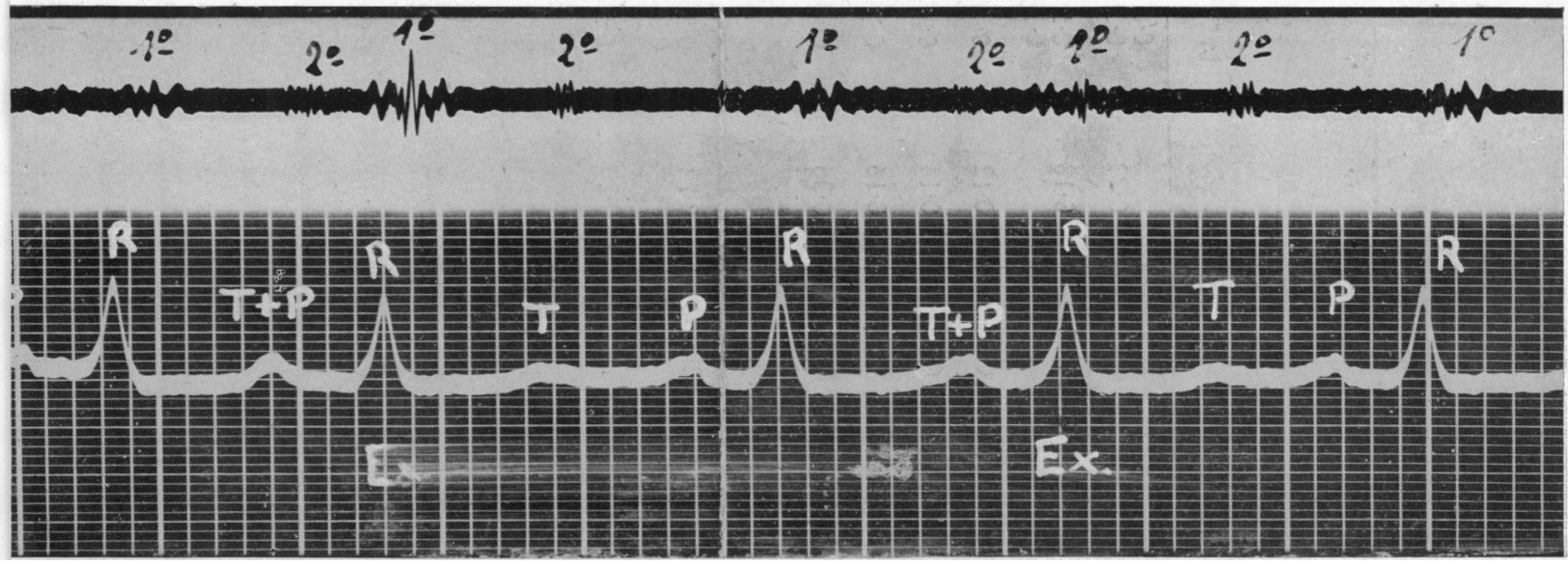

Fig. 3.-Two auricular premature beats are shown: the first occurring in mid-diastole has an intensified and delayed first sound; the second falling later in diastole has a first sound of normal intensity and normal time relation to QRS. 
immediately after the $T$ wave, that is in early diastole, or when it occurred very late in diastole (Fig. 2 and 3). The A-V conduction time in premature beats with an increased first sound appeared to be longer than that of normal beats, but it could not always be determined exactly, as the extrasystolic $\mathbf{P}$ wave was sometimes fused with the preceding $\mathbf{T}$ wave.

\section{VENTRICULAR EXTRASYSTOLES}

In 14 cases investigated (Table II and Fig. 4-7), the extrasystolic first sound was louder than the normal first sound in 9, of equal intensity in 1, of equal or less intensity in 1, and of less intensity in 3 cases. The increased extrasystolic first sound was always delayed in comparison with the normal first sound. The time interval between the onset of QRS and the increased extrasystolic first sound was from 0.08 to 0.12 seconds, average 0.10 seconds, while in the case of normal beats it was 0.03 to 0.06 seconds, average 0.05 seconds. This delay

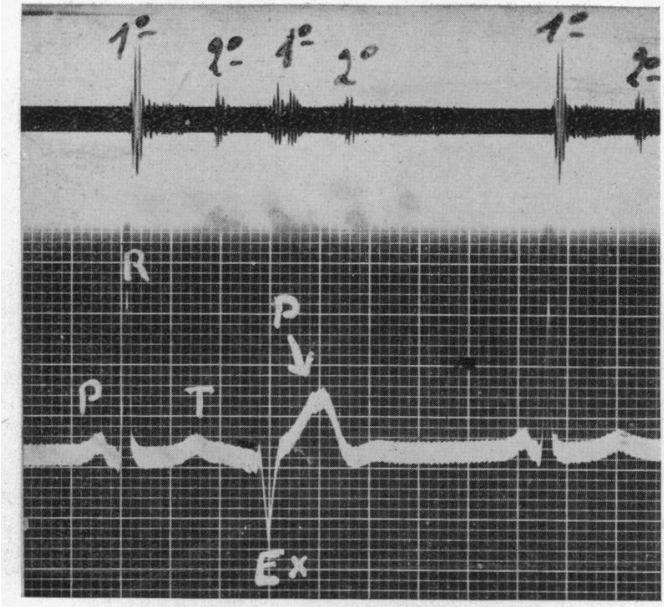

Fig. 4.-Ventricular premature beat falling early in diastole, with faint and reduplicated first sound.

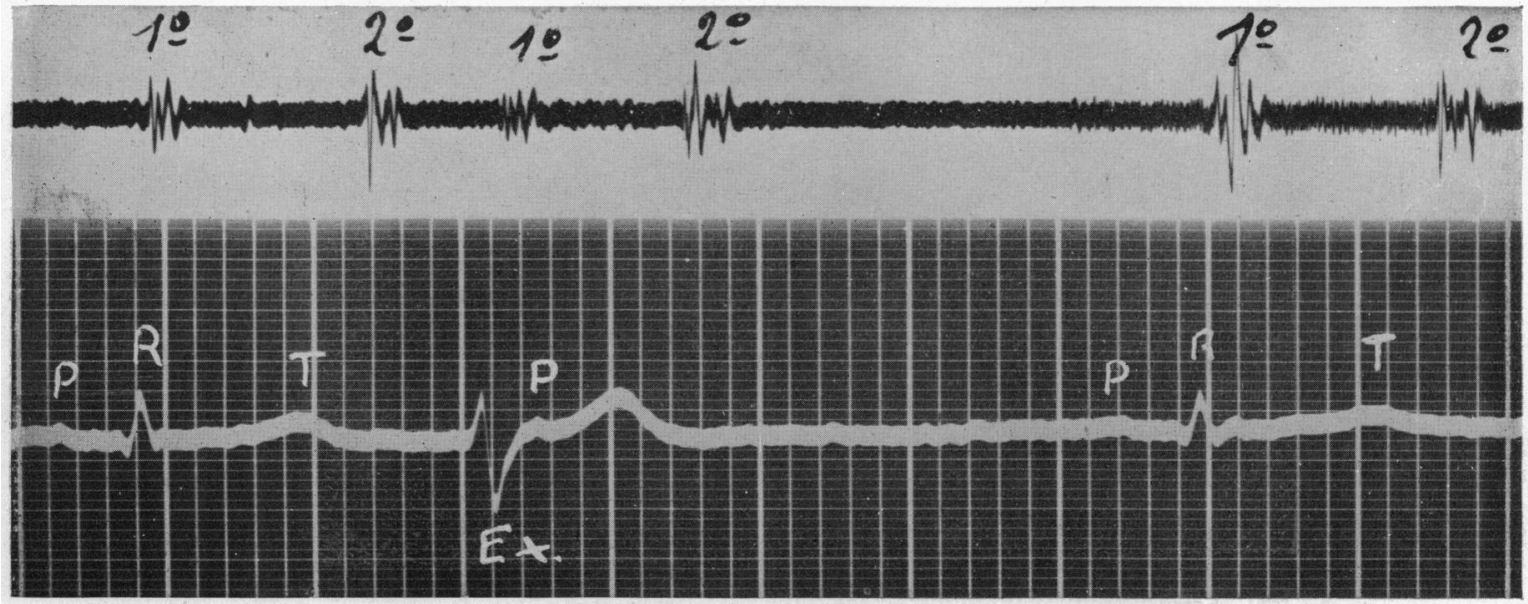

Fig. 5.-Ventricular premature beat falling late in diastole, just before auricular systole, with a faint first sound. 
is especially evident in the case of the group of rapid vibrations of valvular origin belonging to the first sound, but not in the slower inaudible initial vibrations of muscular origin, which when magnified several times, were always found in the same position.

The increased intensity and delay of the first sound in ventricular extrasystoles was related to their degree of prematurity and to their time relation with the normal auricular systole not followed by a ventricular response. Whenever the premature ventricular systole occurred so early that it coincided with the descending limb of the T wave of the preceding cycle (Fig. 4), or fell later just in front of the next $\mathbf{P}$ wave of sinus origin (Fig. 5), the extrasystolic first sound was less intense than that of normal beats. But whenever the premature ventricular systole fell just after the $T$ wave, in mid-diastole (Fig. 6). or just after the next normal $P$ wave (Fig. 7), the extrasystolic first sound was louder than that of normal beats. In 4 of the 14 cases, a split first sound was recorded in the premature beats, though not present in the normal beats.

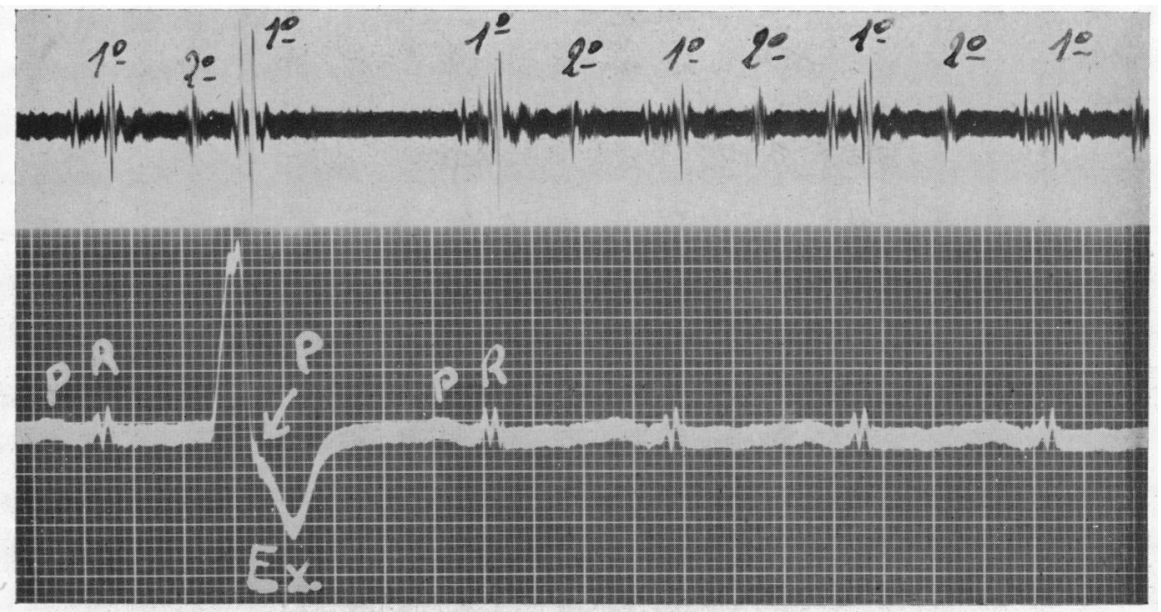

FIG. 6.-Ventricular premature beat, falling, in mid-diastole, with an intensified first sound. Alternation of first sound of succeeding normal beats.

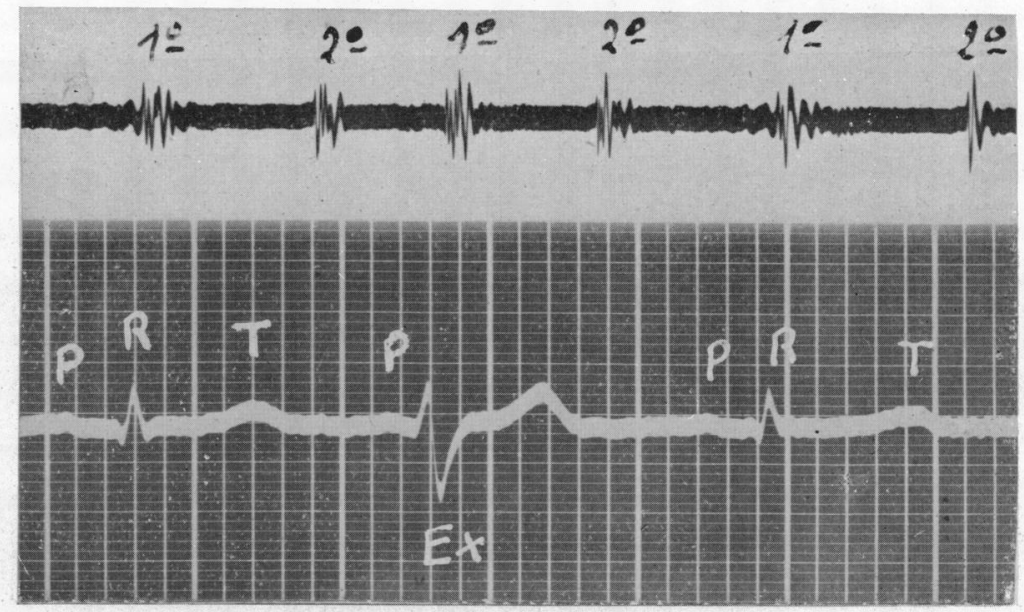

FIG. 7.-Ventricular premature beat falling late in diastole, just after auricular systole, with a slightly intensified and delayed first sound. 


\section{Discussion}

We conclude from our investigations that intensification of the first sound occurs as often in auricular as in ventricular extrasystoles, and, therefore, cannot serve to differentiate between them. A split first sound, on the other hand, is only produced in ventricular extrasystoles, though not in every case, and its presence suggests that an extrasystole is of ventricular origin. Graphic records show that an intensified extrasystolic first sound is always appreciably delayed in relation to the onset of ventricular systole as recorded in the electrocardiogram. This relationship of the intensity of the first sound and the time of its occurrence has been called the law of the first sound (Cossio et al., 1943, 1944, 1945) and it has been attributed to the position and tension of the auriculo-ventricular valves at the onset of systole.

During the cardiac cycle, the A-V valves perform a series of ascending and descending movements with corresponding increase and decrease in their tension. Dean (1936) has shown experimentally that, when blood flows from auricles to ventricles during the phase of rapid inflow, the A-V valves are forced downwards until the maximum opening is attained. As the ventricles fill progressively during diastasis, the A-V valves gradually ascend until the occurrence of auricular systole, when they are again forced downwards, ascending again to a position of almost complete closure on the termination of auricular systole. Normally, the ventricular contraction finds the valves in this position, and their maximum stretch follows immediately; the vibration so produced, which Dock (1933) has shown to be the fundamental cause of the first sound, practically coincides with the onset of ventricular systole.

But, if a ventricular contraction happens to occur at some other moment, for example, at the end of the phase of rapid inflow or during auricular systole, it will find the A-V valves in a much lower position, so that the distance travelled to reach the position of maximum stretch is longer, and consequently more time elapses between the onset of ventricular systole and the valvular vibration that gives rise to the first sound. When the valves have further to travel between the onset of ventricular contraction and the position of maximum stretch, their movement is accelerated, and consequently their vibration is increased, for the kinetic energy is the product of half the mass and the square of the velocity, and the velocity is the product of acceleration and time. This is precisely what has been shown to occur in premature beats, both auricular and ventricular, though more frequently in the former than the latter. When the onset of ventricular systole in a premature beat coincides with an auricular systole of sinus origin, the first sound is intensified and delayed. Similarly, when the ventricular systole of either an auricular or ventricular premature beat falls at the end of or immediately after the phase of rapid inflow, the first sound is intensified and delayed.

Thus there are two propitious moments for the production of the intensified and delayed first sound, at both of which the A-V valves are in their lowest position. When a premature beat falls before the end of rapid inflow, the A-V valves are also lowered, but in this case, owing to incomplete ventricular filling, the valves are insufficiently stretched to intensify the first sound.

There are several reasons why the first sound is more often intensified and delayed in auricular than in ventricular extrasystoles. The first sound represents the summation of vibrations produced by the mitral and tricuspid valves. In auricular extrasystoles this normal summation is unaffected, but in ventricular extrasystoles a synchronism between the two ventricles prevents summation, and the first sound is split. Another reason is that auricular extrasystoles generally fall at a moment propitious for intensification of the first sound. Lastly, ventricular filling is more complete in auricular than in ventricular extrasystoles, because the former have the benefit of an auricular contraction which also contributes to lowering the A-V valves.

\section{SUMMARY}

The characteristics of the first heart sound in auricular and ventricular premature beats 
have been investigated by phonocardiographic records from 30 cases. In both varieties of extrasystole, the first sound was usually increased in intensity and delayed in relation to the onset of ventricular systole. In ventricular premature beats the first sound was sometimes split. The explanation of these findings is discussed in relation to the position and movements of the auriculo-ventricular valves.

\section{REFERENCES}

Cossio, P. (1944). Semiologia Aparato Circulatoria. 4th ed., Buenos Aires. P. (1943). Rev. Argent. Cardiol., 10, 145. and Berconsky, I. (1943). Ibid., 10, 163. (1943). Ibid., 10, 283. and Trimani, A. (1944). Ibid., 10, 363 __ and Sotomayor, O. (1945). Ibid., 12, 70. Dean, A. L. (1916). Amer. J. Physiol., 40, 213. Dock, W. (1933). Ibid., 51, 737.

Lewis, T. (1935). Clinical Disorders of the Heart Beat. 5th ed., London. 